\title{
Separate freeze-out of strange particles and the quark-hadron phase transition
}

\author{
K. Bugaev ${ }^{1, a}$, V. Sagun ${ }^{1,2}$, A. Ivanytskyi ${ }^{1}$, E. Nikonov ${ }^{3}$, J. Cleymans ${ }^{4}$, I. Mishustin ${ }^{5,6}$, G. Zinovjev $^{1}$, \\ L. V. Bravina ${ }^{7}$ and E. E. Zabrodin ${ }^{7,8,9}$ \\ ${ }^{1}$ Bogolyubov Institute for Theoretical Physics, Metrologichna str. 14 b, 03143 Kyiv, Ukraine \\ ${ }^{2}$ Centro de Astrofísica e Gravitação - CENTRA, Departamento de Física, Instituto Superior Técnico - IST, \\ Universidade de Lisboa - UL, Av. Rovisco Pais 1, 1049-001 Lisboa, Portugal \\ ${ }^{3}$ Laboratory for Information Technologies, JINR, Joliot-Curie str. 6, 141980 Dubna, Russia \\ ${ }^{4}$ Department of Physics, University of Cape Town, Rondebosch 7701, South Africa \\ ${ }^{5}$ FIAS, Goethe-University, Ruth-Moufang Str. 1, 60438 Frankfurt upon Main, Germany \\ ${ }^{6}$ Kurchatov Institute, Russian Research Center, Kurchatov Sqr., Moscow, 123182, Russia \\ ${ }^{7}$ Department of Physics, University of Oslo, PB 1048 Blindern, N-0316 Oslo, Norway \\ ${ }^{8}$ Skobeltzyn Institute of Nuclear Physics, Moscow State University, 119899 Moscow, Russia \\ ${ }^{9}$ National Research Nuclear University "MEPhl" (Moscow Engineering Physics Institute), Kashirskoe Shosse \\ 31, 115409 Moscow, Russia
}

\begin{abstract}
The scenario of the independent chemical freeze-outs for strange and nonstrange particles is discussed. Within such a scenario an apparent in-equilibrium of strangeness is naturally explained by a separation of chemical freeze-out of strange hadrons from the one of non-strange hadrons, which, nevertheless, are connected by the conservation laws of entropy, baryonic charge and third isospin projection. An interplay between the separate freeze-out of strangeness and its residual non-equilibrium is studied within an elaborate version of the hadron resonance gas model. The developed model enables us to perform a high-quality fit of the hadron multiplicity ratios measured at AGS, SPS and RHIC with an overall fit quality $\chi^{2} / d o f=0.93$. A special attention is paid to a description of the Strangeness Horn and to the well-known problem of selective suppression of $\Lambda^{-}$and $\Xi$ hyperons. It is remarkable that for all collision energies the strangeness suppression factor $\gamma_{s}$ is about 1 within the error bars. The only exception is found in the vicinity of the center-of-mass collision energy $7.6 \mathrm{GeV}$, at which a residual enhancement of strangeness of about $20 \%$ is observed.
\end{abstract}

\section{Introduction}

Evolution of strongly interacting system created in high energy central heavy ion collisions includes several stages governed by different physical degrees of freedom. Switching between them is expected to have a character of a phase transition. For an unambiguous identification of these transitions of QCD we need the reliable signals which can be measured experimentally. However, an interpretation of such signals is highly non-trivial, since in the region of major interest the lattice formulation of QCD

\footnotetext{
a e-mail: bugaev@fias.uni-frankfurt.de
} 
is inapplicable due to high baryonic charge densities. The problems is enhanced because most of such signals are affected by the last stage of nucleus-nucleus collision, at which the strongly interacting matter exists in the form of a hadron gas. Therefore, one of the most important theoretical tasks is to separate or to account for an influence of the hadron gas expansion from true signals of phase transformation. Evidently, to solve this task one needs a reliable theoretical description of hadron gas properties emitted from the hypersurface of chemical freeze-out [1-4].

During last four years such an approach known as the hadron-resonance gas model (HRGM) [14] was worked out. Up to now, the HRGM is the most successful approach to describe the yields of produced hadrons. However, we have to point out that the obsolete versions of this model which have only one or two independent hadronic hard core radii were not able to give a high quality description of available experimental data. The true progress in obtaining an accurate description of the data was recently achieved by the multicomponent version of the HRGM (MHRGM) [3]. Thus, having only two additional (global) fitting parameters the high quality description of the most problematic ratios of hadrons was achieved $[3,4]$. In the works $[3,4]$ the ratios $K^{+} / \pi^{+}$and $\Lambda / \pi^{-}$were described with the quality $\chi_{K^{+} / \pi^{+}}^{2} / d o f=\frac{7.7}{14}$ and $\chi_{\Lambda / \pi^{-}}^{2} / d o f=\frac{16}{12}$ which is a perfect description compared to the results $\chi_{K^{+} / \pi^{+}}^{2} / d o f=\frac{21.8}{14}$ and $\chi_{\Lambda / \pi^{-}}^{2} / d o f=\frac{79}{12}$ obtained in Ref. [5]. Unfortunately, even this advanced model failed to reproduce the yields of strange particles measured at several collision energies. Therefore, one cannot use such a model to reliably study the irregularities associated to strange hadrons at chemical freeze-out. It is clear that without accurate fit of the data one cannot draw any reliable conclusion about such irregularities. Therefore, in order to improve the description of strange particle yields in Ref. [6] it was assumed that strange hadrons can deviate from the full chemical equilibrium. The degree of such a deviation from chemical equilibrium is controlled by the $\gamma_{s}$ factor [6], which modifies the one-particle thermal density $\phi_{i}$ of strange hadron of sort $i$ according to the rule

$$
\phi_{i} \rightarrow \gamma_{s}^{s_{i}} \phi_{i}
$$

Here $s_{i}$ denotes the total number of strange valence quarks and antiquarks in a given hadron. If $\gamma_{s}=1$, then there is no deviation of strange particles from chemical equilibrium. Their production is enhanced, if $\gamma_{s}>1$, and is suppressed, if $\gamma_{s}<1$. Being treated as a free parameter, the $\gamma_{s}$ factor, indeed, sizably improves the description of particle yields within the MHRGM and provides an overall fit with $\chi^{2} / d o f=1.15$ in case of four independent hard-core radii of hadrons [8] and with $\chi^{2} / d o f=0.95$, if the number of these radii is five $[4,9]$. However, a physical reason lying behind the deviation of strange charge from chemical equilibrium remains unclear. In order to elucidate this reason here we consider several scenaria of two independent freeze-outs, which correspond to nonstrange and strange hadrons, which, nevertheless, are connected by the conservation laws. Due to the latter feature this approach significantly improves the quality of experimental data description.

In the next section we briefly formulate the MHRGM and introduce the concept of separate freezeout of strange particles (SFO) from the freeze-out of non-strange hadrons (FO). Analysis of experimental data is presented in Section 3. The question about the source of residual in-equilibrium of strange particles is also discussed in this section. Finally, our conclusions are given in Section 4.

\section{Separate freeze-out of strange particles}

The MHRGM model treats hadron gas as a multicomponent mixture of hard spheres with the hard core radii $R_{i}$ with $i=1,2,3, \ldots$. The grand canonical pressure $p$ of such a mixture is a sum of hadronic partial pressures $p_{i}$ defined as a solution of the following nonlinear system

$$
p_{i}=\phi_{i} \exp \left[\frac{\mu_{i}-2 \sum_{j} b_{i j} p_{j}+p^{-1} \sum_{j k} p_{k} b_{k j} p_{j}}{T}\right] \text {. }
$$


Here $T$ denotes the temperature, while $\mu_{i}=\mu^{B} Q_{i}^{B}+\mu^{I 3} Q_{i}^{I 3}+\mu^{S} Q_{i}^{S}$ is the chemical potential of a given hadron sort, which is expressed in terms of the baryonic $\mu_{B}$, the third isospin projection $\mu_{I 3}$ and strange $\mu_{S}$ chemical potentials associated with the corresponding charges $Q_{i}^{B}, Q_{i}^{I 3}$ and $Q_{i}^{S}$ of this hadron, respectively. A symmetric matrix $b_{i j}=\frac{4}{3} \pi\left(R_{i}+R_{j}\right)^{3}$ is composed from the second virial coefficients of different hadron species. The one particle thermal density $\phi_{i}$ of a hadron with the spin-isospin degeneracy $g_{i}$ and the mean mass $m_{i}$ is defined as the momentum integral of the Boltzmann exponential, which is averaged over the normalized Breit-Wigner mass distribution $f_{B W}$ with the threshold $m_{i}^{T h}$ and the width $\Gamma_{i}$

$$
\phi_{i}=g_{i} \int_{m_{i}^{T h}}^{\infty} d m f_{B W}\left(m_{i}, \Gamma_{i}, m\right) \int \frac{d^{3} k}{(2 \pi)^{3}} \exp \left[-\frac{\sqrt{k^{2}+m^{2}}}{T}\right], f_{B W}\left(m_{i}, \Gamma_{i}, m\right)=\frac{\Gamma_{i}\left[N_{i}\left(m_{i}^{T h}\right)\right]^{-1}}{\left(m-m_{i}\right)^{2}+\Gamma_{k}^{i} / 4},
$$

where the normalization fact is defined as $N_{i}\left(m_{i}^{T h}\right) \equiv \int_{m_{i}^{T h}}^{\infty} \frac{d m \Gamma_{i}}{\left(m-m_{i}\right)^{2}+\Gamma_{i}^{2} / 4}$. If all partial pressures $p_{i}$ are known, then the thermal densities of hadrons are calculated by a differentiation of $p$ with respect to its chemical potential, i.e. $n_{i}=\frac{\partial p}{\partial \mu_{i}}$.

Contrary to the conventional approach, the present model allows one to freeze-out the multiplicities of strange and non-strange hadrons independently from each other. As a result, the thermodynamic parameters of FO and SFO are different in general case. These ten parameters are the temperatures $T_{A}$, the baryonic $\mu_{A}^{B}$, the strange $\mu_{A}^{S}$ and the third isospin projection $\mu_{A}^{I 3}$ chemical potentials defined for $A \in\{F O, S F O\}$ as well as the volumes of the FO and SFO hypersurfaces, respectively, $V_{F O}$ and $V_{S F O}$. However, a hydrodynamic evolution of the system between these two hypersurfaces of heavy ion collisions implies the conservation of entropy. The other conserved quantities are the baryonic and electric charges. Therefore, we assume that

$$
s_{F O} V_{F O}=s_{S F O} V_{S F O}, \quad n_{F O}^{B} V_{F O}=n_{F O}^{B} V_{S F O}, \quad n_{F O}^{I 3} V_{F O}=n_{F O}^{I 3} V_{S F O},
$$

where $s_{A}=\frac{\partial p}{\partial T}, n_{A}^{B}=\frac{\partial p}{\partial \mu_{A}^{B}}$ and $n_{A}^{I 3}=\frac{\partial p}{\partial \mu_{A}^{I 3}}$ denote the total densities of entropy, baryonic charge and the third isospin projection at FO or SFO. These equations allow us to exclude three parameters of SFO, i.e. $V_{S F O}, \mu_{S F O}^{B}$ and $\mu_{S F O}^{I 3}$, from the list of independent ones. Two more parameters, namely the strange chemical potentials $\mu_{F O}^{S}$ and $\mu_{S F O}^{S}$, are excluded by the requirement of zero total strangeness at FO and SFO. Thus, only five parameters remains independent within our approach. They are $T_{F O}, T_{S F O}$, $\mu_{F O}^{B}, \mu_{F O}^{I 3}$ and $V_{F O}$. In other words, in the present model the number of independent fitting parameters for each collision energy is exactly the same as in the HRGM with the $\gamma_{s}$ factor, but instead of the latter one, the parameter $T_{S F O}$ is used.

The strong decays of hadronic resonances modify their multiplicities, while the weak ones can be safely neglected in the considered range of collision energies. Thus, the total number of produced hadrons of sort $i$ is calculated as

$$
N_{i}^{t o t}=V_{F O} \sum_{j \in F O} B r(j \rightarrow i) n_{j}+V_{S F O} \sum_{j \in S F O} B r(j \rightarrow i) n_{j} .
$$

Here the branching ratio $\operatorname{Br}(j \rightarrow i)$ defines a probability that the hadron of sort $j$ decays into a hadron of sort $i$ and $\operatorname{Br}(i \rightarrow i)=1$ is introduced for the sake of convenience. In order to get rid of effective volume of the system $V_{F O}$ at FO we fitted the particle number ratios $R_{i j} \equiv \frac{N_{i}^{\text {tot }}}{N_{j}^{\text {tot }}}$ instead of their total multiplicities. The conservation laws (4) in this case are written in the form which does not contain the effective volumes

$$
\frac{s_{F O}}{n_{F O}^{B}}=\frac{s_{S F O}}{n_{S F O}^{B}}, \quad \frac{n_{F O}^{I 3}}{n_{F O}^{B}}=\frac{n_{S F O}^{I 3}}{n_{S F O}^{B}} .
$$



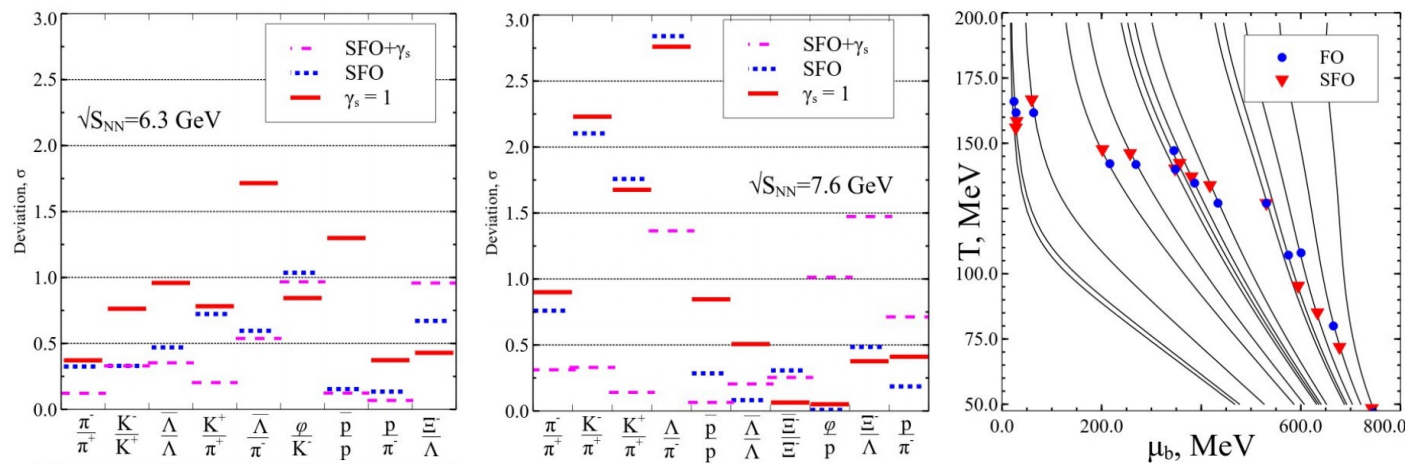

Figure 1. Left panel. Deviations of theoretical particle yield ratios from their experimental values in units of experimental errors at $\sqrt{s_{N N}}=6.3 \mathrm{AGeV}$. Calculations are performed by the MHRGM for the combined fir of separate SFO and $\gamma_{s}$ (dashed bars), for the SFO only (dotted bars) and without both of them (solid bars). Middle panel. The same as in the left panel but for $\sqrt{s_{N N}}=7.6 \mathrm{AGeV}$. Right panel. Points of FO (circles) and SFO (triangles) obtained from the particle multiplicities fit performed within the MHRGM with two separate freezeouts and without $\gamma_{s}$. The solid curves represent the adiabats $s / n_{B}=$ const which correspond to the continuous hydrodynamic evolution of the system.

The masses of hadrons, their spin-isospin degeneracies, the widths, as well as the branching ratios and thresholds of strong decays, are taken from the particle tables used by the thermodynamic code THERMUS [7]. The hard core radii of hadrons were fixed to the best fit values from Refs. [3, 8].

\section{Analysis of experimental data}

The experimental data set used in our analysis [3, 4, 8-10] follows the one of Ref. [2]. We fitted the ratios of particle multiplicities measured at midrapidity at 14 different collision energies in the collision energy interval from $\sqrt{s_{N N}}=2.7-200 \mathrm{AGeV}$. At low AGS energies $E_{l a b}=2,4,6,8 \mathrm{AGeV}$ data for pions [11, 12], protons [13, 14] and kaons [11] (except for $2 \mathrm{AGeV}$ ) are available with high resolution. Instead of integrated over $4 \pi$ raw experimental data measured for $\Lambda$ hyperons at $2-8$ AGeV [15] and for $\Xi^{-}$at $6 \mathrm{AGeV}$ [16] we used an accurately corrected values from Ref. [2]. At $\sqrt{s_{N N}}=4.9 \mathrm{AGeV}\left(E_{l a b}=10.7 \mathrm{AGeV}\right)$ we used only the NA49 data at midrapidity [17-22]. The RHIC data at $\sqrt{s_{N N}}=9.2 \mathrm{AGeV}[23], \sqrt{s_{N N}}=62.4 \mathrm{AGeV}$ [24], $\sqrt{s_{N N}}=130 \mathrm{AGeV}$ [25-28] and $\sqrt{s_{N N}}=200 \mathrm{AGeV}[28-30]$ were taken from the STAR collaboration.

The fit procedure to find out the parameters $T_{F O}, T_{S F O}, \mu_{F O}^{B}$ and $\mu_{F O}^{I 3}$ at each collision energy searches for a global minimum of the quantity

$$
\chi^{2}=\sum_{i j}\left(\frac{R_{i j}^{\text {theor }}-R_{i j}^{\text {exp }}}{\delta R_{i j}^{\text {exp }}}\right)^{2},
$$

where summation is performed over all independent pairs of particle sorts $i$ and $j$, while the superscript indices "theor" and "exp" denote, respectively, theoretically calculated and experimentally measured values of the corresponding ratio $R_{i j}$ which has the experimental errors $\delta R_{i j}^{\exp }$.

The two main subjects of our analysis are the values of thermodynamic parameters at chemical freeze-out and what ratios are improved by introduction of separate SFO. From the fit results we 

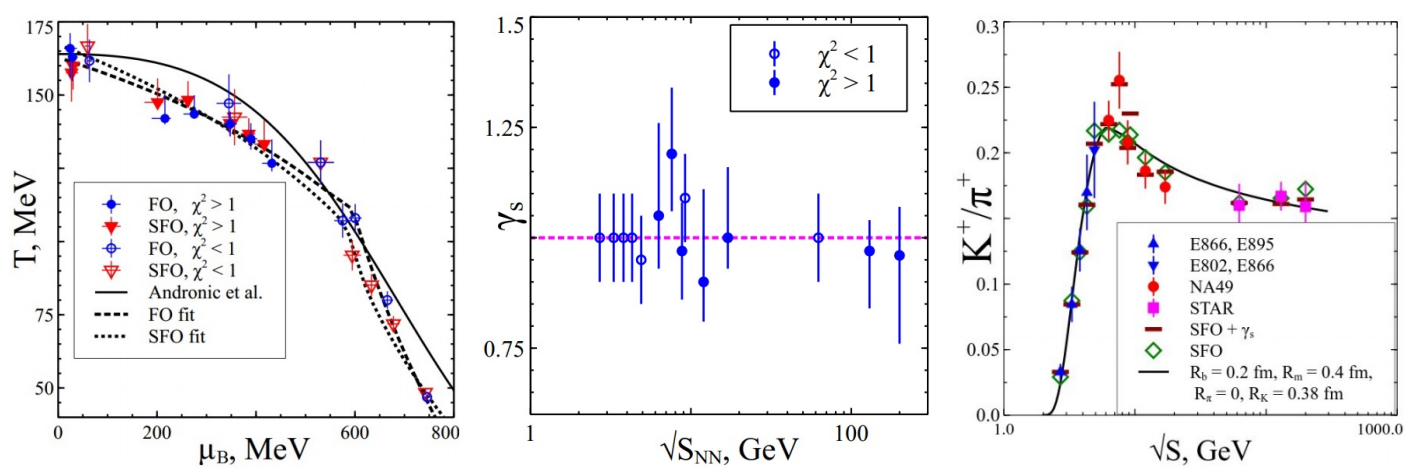

Figure 2. Left panel. Points of SFO and FO in the plane of baryonic chemical potential $\mu_{B}$ and temperature $T$. The solid curve represents a parameterization of Ref. [2], the dashed and short dashed curves correspond to parameterizations from Ref. [31]. Middle panel. Dependence of the $\gamma_{s}$ factor on the collision energy from the combined fit (see text). Right panel. Collision energy dependence of the ratio $K^{+} / \pi^{+}$. Horizontal bars and empty diamonds represent the results obtained for the combined fit and for the SFO fit, respectively. The solid curve represents the parameterization of baryonic chemical potential and temperature taken from Ref. [31].

conclude that traditionally problematic ratios which involve the strange particles experience the most significant influence due to separate SFO. This effect is stronger at the intermediate collision energies. For example, at $\sqrt{s_{N N}}=6.3 \mathrm{AGeV}$ an introduction of SFO makes the ratios $K^{-} / K^{+}, \bar{\Lambda} / \Lambda$ and $\bar{\Lambda} / \pi^{-}$ much closer to their experimental values (see the left panel of Fig. 1). It is worth to stress, that the description of another problematic ratio $\bar{p} / p$ is also greatly improved within the MHRGM with separate SFO. This means that the present approach relaxes a connection between strange and nonstrange baryons and significantly simplifies their simultaneous description. As it is seen from the middle panel of Fig. 1 the separate SFO approach sizably improves a description of the $\mathrm{K}^{-} / \pi^{+}$, $K^{+} / \pi^{+} \Lambda / \pi^{-}, \bar{p} / p$ and $\bar{\Lambda} / \Lambda$ ratios at $\sqrt{s_{N N}}=7.6 \mathrm{AGeV}$. Since, the highest sensitivity of the particle yield ratios on the SFO parameters is achieved at $\sqrt{s_{N N}}$ from 6.3 to $17 \mathrm{AGeV}$, then there is nothing unexpected that separation of the curves of two freeze-outs is the most pronounced in this region. Such a conclusion can be drawn from the right panel of Fig. 1, where the curves of SFO and FO are shown in the plane of baryonic chemical potential and temperature. This figure shows that these curves are rather close to each other. Although the difference between $T_{S F O}\left(\sqrt{s_{N N}}\right)$ and $T_{F O}\left(\sqrt{s_{N N}}\right)$ does not exceed $20 \mathrm{MeV}$, it is sufficient for a systematic improvement of the data description reflected in the overall value of $\chi^{2} /$ dof $=58.5 / 55 \simeq 1.06$ [8].

It is remarkable that introduction of separate SFO reduces the $\chi^{2} /$ dof value by about $10 \%$ from initial value 1.16 obtained in Ref. [3]. At the same time the MHRGM without SFO and with the free $\gamma_{s}$ parameter allows one to describe the data with overall value of $\chi^{2} / d o f=1.15$ which is only slightly less than the result of Ref. [3]. This means that the scenario of separate SFO is favored by experimental data. Moreover, the concept of separate SFO naturally explains an apparent chemical in-equilibrium of strange hadrons used to heal the inconsistency between the experimental and theoretical particle ratios. Indeed, if the SFO is ignored, then the multiplicities of strange hadrons are assumed to be controlled by the temperature and chemical potentials of FO which, however, are not the equilibrium parameters of these particles and can not provide the high quality description of their yields. This effect looks like a deviation of strangeness from chemical equilibrium. Thus, a poor description of yields of strange hadrons obtained in the earlier versions of HRGM can not be 
treated as an unambiguous evidence of chemical in-equilibrium of these particles, whereas the parameter $\gamma_{s}$ is likely an extra parameter of thermal models. At the same time, usage of of this factor is justified from the practical point view, since it provides an opportunity to describe the $K^{+} / \pi^{+}$ratio with $\chi_{K^{+} / \pi^{+}}^{2} / d o f=3.3 / 14$. This result is even better than $\chi_{K^{+} / \pi^{+}}^{2} / d o f=6.3 / 14$ obtained within the MHRGM with separate SFO. Since the $\gamma_{s}$ approach provides an exceptional description of the Strangeness Horn, whereas the SFO framework allows one to obtain remarkably good results for all other hadrons, then a combination of these two methods seems to be natural in order to reach the highest quality of the experimental data description. Such a combined fit is also important since it can serve as an a posteriori check of the fact that there exists a residual chemical in-equilibrium of strange particles. The MHRGM with separate SFO and free $\gamma_{s}$ was used to analyze the particle yields in Ref. [31]. As it is seen from Fig. 1 the description of data in this case is even better than within the MHRGM with the separate SFO alone. A high quality of the fit is reflected in the overall value of $\chi^{2} / d o f \simeq 0.93$ which is the best result obtained up to now. Our special attention was paid to the behavior of the SFO and FO parameters. Similarly to the results of Ref. [8] the curves of two freezeouts are located quite close to each other in the plane of baryonic chemical potential and temperature (see the left panel of Fig. 2). One of the most important conclusions of the combined fit is that $\gamma_{s}=1$ within error bars for all collision energies except for 6.3 and $7.6 \mathrm{AGeV}$, which maybe related to the chiral symmetry restoration in the non-strange sector and its absence in the strange one [32, 33] at these energies. It is also interesting that in this region the strange hadrons are enhanced due to $\gamma_{s}>1$. This is clearly seen from the middle panel of Fig. 2. From this finding we conclude that an apparent deviation of strange hadrons from chemical equilibrium is just a small residual effect of SFO. As it is seen from the right panel of Fig. 2, even the topmost point of the Strangeness Horn is reproduced well within our approach. The corresponding value of $\chi_{K^{+} / \pi^{+}}^{2} / d o f$ is only $1.5 / 14$ which evidences for the best description of this problematic ratio.

\section{Conclusions}

Based on the realistic equation of state which is suitable for to describe the multicomponent mixtures with the hard core repulsion between the constituents we developed a novel version of the HRGM. Introduction of the separate SFO into this model allowed us to perform a reliable study of hadronic matter thermodynamics at chemical freeze-out and to describe the hadronic yield ratios with the highest quality ever achieved. Moreover, it is shown that an apparent in-equilibrium of strange hadrons can be naturally explained by the concept of separate SFO, while the residual in-equilibrium is small. Nevertheless, due to the strong sensitivity of the $\gamma_{s}$ factor to phase transformations in QCD [32, 33], we believe it is worth to use it as a special indicator of such transformations.

Acknowledgments: KAB, A.I.I., V.V.S. and G.M.Z. acknowledge a partial support from the Program of Fundamental Research of the Department of Physics and Astronomy of National Academy of Sciences of Ukraine. L. V. B. and K. A. B. thank the Norwegian Centre for International Cooperation in Education (SIU) for financial support, grant "CPEA-LT-2016/10094 From Strong Interacting Matter to Dark Matter". L. V. B., K. A. B and E.E.Z. are thankful to Norwegian Research Council for financial support, grant "255253/F50 - CERN Heavy Ion Theory" V.V.S. thanks the Fundação para a Ciência e Tecnologia (FCT), Portugal, for the financial support to make her research at the Centro de Astrofísica e Gravitação (CENTRA), Instituto Superior Técnico, Universidade de Lisboa.

\section{References}

[1] P. Braun-Munzinger, K. Redlich, J. Stachel, arXiv:nuclth/0304013v1. 
[2] A. Andronic, P. Braun-Munzinger, J. Stachel, Nucl. Phys. A 772, 167 (2006) and references therein.

[3] K. A. Bugaev, D. R. Oliinychenko, A. S. Sorin, G. M. Zinovjev, Eur. Phys. J. A 49, 30 (2013).

[4] V. V. Sagun, Ukr. Phys. J., 59, 8, 755 (2014).

[5] A. Andronic, P. Braun-Munzinger, J. Stachel, Phys. Lett. B 673, 142 (2009).

[6] J. Rafelsky, Phys. Lett. B 62, 333 (1991).

[7] S. Wheaton, J. Cleymans and M. Hauer, Comput. Phys. Commun. 180, 84 (2009).

[8] K. A. Bugaev et al., Europhys. Lett. 104, 22002 (2013).

[9] V. V. Sagun et al., Ukr. Phys. J., 59, 11, 1043 (2014).

[10] K. A. Bugaev et al., Ukr. J. Phys., 61, 8, 659 (2016).

[11] L. Ahle et al., Phys. Lett. B 476, 1 (2000).

[12] J. L. Klay et al., Phys. Rev. C 68, 054905 (2003).

[13] B. B. Back et al., Phys. Rev. Lett. 86, 1970 (2001).

[14] L. Klay et al., Phys. Rev. Lett. 88, 102301 (2002).

[15] C. Pinkenburg et al., Nucl. Phys. A 698, 495 (2002).

[16] P. Chung et al., Phys. Rev. Lett. 91, 202301 (2003).

[17] S. V. Afanasiev et al., Phys. Rev. C 66, 054902 (2002).

[18] S. V. Afanasiev et al., Phys. Rev. C 69, 024902 (2004).

[19] T. Anticic et al., Phys. Rev. Lett. 93, 022302 (2004).

[20] S. V. Afanasiev et al., Phys. Lett. B 538, 275 (2002).

[21] C. Alt et al., Phys. Rev. Lett. 94, 192301 (2005).

[22] S.V. Afanasiev et al., Phys. Lett. B 491, 59 (2000).

[23] B. Abelev et al., Phys. Rev. C 81, 024911 (2010).

[24] B. Abelev et al., Phys. Rev. C 79, 034909 (2009).

[25] J. Adams et al., Phys. Rev. Lett. 92, 182301 (2004).

[26] J. Adams et al., Phys. Lett. B 567, 167 (2003).

[27] C. Adler et al., Phys. Rev. C 65, 041901(R) (2002).

[28] J. Adams et al., Phys. Rev. Lett. 92, 112301 (2004).

[29] J. Adams et al., Phys. Lett. B 612, (2005) 181.

[30] A. Billmeier et al., J. Phys. G 30, S363 (2004).

[31] K. A. Bugaev et al., Ukr. J. Phys., 62, 659 (2016).

[32] K. A. Bugaev et al., arXiv:1709.05419 [hep-ph] (accepted to PEPAN Lett.).

[33] K. A. Bugaev et al., arXiv:1711.07283 [nucl-th]. 